\title{
Pressure Influence on the Curie Temperature
}

\author{
J. RutKowski ${ }^{a}$, L. WOJTCZAK ${ }^{a}$ AND Š. ZAJAC ${ }^{b}$ \\ ${ }^{a}$ Department of Solid State Physics, University of Łódź, Pomorska 149/153, 90-236 Łódź, Poland \\ ${ }^{b}$ Faculty of Nuclear Sciences and Physical Engineering, Czech Technical University, Prague, Czech Republic \\ The pressure dependence of the ferromagnetic-paramagnetic phase transition temperature $T_{\mathrm{C}}(p)$ is of high \\ interest due to its direct technological implications. The theoretical investigations of the Curie temperature $T_{\mathrm{C}}(p)$ \\ considered in the ferromagnetic crystals have been studied employing various methods of calculations. The present \\ paper is devoted to its description by means of the pseudoharmonic approximation approach.
}

PACS numbers: 75.30.Kz, 75.40.-s, 75.50.Cc, 81.40.Vw, 64.30.Ef

\section{Introduction}

The present paper deals with the pressure influence on the Curie temperature in ferromagnets whose effective potential of the lattice is described by the modified Morse potential $U\left(R_{r}-R_{r^{\prime}}\right)$ given in terms of pseudoharmonic approximations [1]. The pressure $p$ is applied in the form of isotropic (hydrostatic) external force to the whole sample and scaled with respect to the normal (atmospheric) pressure $p_{0}\left(p_{0}=1.0135 \times 10^{-4} \mathrm{GPa}\right)$.

Magnetic properties are discussed at the level of the localized spins model. The magnetic moments are situated in the lattice sites whose distance expands or compresses due to the thermal vibrations or the pressure compression. The exchange integral $J\left(R_{r}-R_{r^{\prime}}\right)$ as well as the potential $U\left(R_{r}-R_{r^{\prime}}\right)$ change their values due to the distance $R_{r}-R_{r^{\prime}}=R$ between two localized spins where $R$ is the equilibrium position at a given temperature $T$ under a given pressure $p: R=R(p, T)$; in particular $R_{0}=R\left(p_{0}, T=0\right)$.

We assume that the Curie temperature is proportional to the exchange integral $J(R)$ calculated by means of the effective distance between two localized spins situated at $r$ and $r^{\prime}$. The pressure effect is introduced then by the equation of state $R=R\left(p, T_{\mathrm{C}}(p)\right)$. The shape of the relation $J=J(R)$ is determined by the analogy to the case when we consider the random distribution of exchange integrals for the samples with the amorphous structure [2].

In the methodological context the paper is a continuation of the melting temperature $T_{\mathrm{m}}(p)$ calculations [3] while the essential problem is connected with the considerations concerning the Curie temperature and its dependence on the pressure $[4,5]$.

\section{The outline of the model}

The effective Hamiltonian contains three terms corresponding to the lattice and magnetic properties, namely

$$
\begin{aligned}
H & =\frac{1}{2} \sum_{r} \frac{P_{r}^{2}}{M}+\frac{1}{2} \sum_{r r^{\prime}} U\left(R_{r}-R_{r^{\prime}}\right) \\
& -\sum_{r r^{\prime}} J\left(R_{r}-R_{r^{\prime}}\right) S_{r}^{z} S_{r^{\prime}}^{z},
\end{aligned}
$$

where $r, r^{\prime}$ stand for the sites occupied by atoms with spins $S_{r} ; P_{r}$ stand for impulse momenta whose mass is denoted by $M$.
The coupling between phonons and magnons appears by means of the dependence of the exchange integral on the effective distance between the positions of two localized spins which are situated in the effective lattice sites, so that the geometry of crystallographic structure and magnetic network is the same. The pseudoharmonic approach corresponds to the description of the temperature renormalization of the equation of state in terms of the mean square displacements of atoms from their positions of equilibrium. The magnetic properties are described by the Ising model while the lattice properties are considered in the lattice potential which takes into account the influence of the pressure due to the additional term $p V$ where $V \sim R^{3}$ stands for the elementary cell volume $V=V(p, T(p))$.

From the physical point of view the method of pseudoharmonic approximation is equivalent to the averaging of the free energy with respect to the Gaussian distribution which is characterized by the mean square displacement of atoms from their equilibrium positions: $y=y(p, T)$.

In the case of the Morse potential the pseudoharmonic procedure leads to the equation of state (see, e.g. [1]): $r^{2} \mathrm{e}^{2 y}-r \mathrm{e}^{y / 2}=p^{*} \omega^{2}$ where

$$
\begin{aligned}
& r=\mathrm{e}^{-6\left(\frac{R}{R_{0}}-1\right)}, \quad \omega=\frac{R}{R_{0}}, \quad p^{*}=\left(\frac{p}{p_{0}}-1\right) \Gamma, \\
& \Gamma=\left(\frac{p_{0} V_{0}}{4 D}\right)
\end{aligned}
$$

with the coefficient $\Gamma$ interpreted as the material constant and the parameter

$$
y(p, T)=\frac{T}{T_{\mathrm{m}}}\left\{\mathrm{e}\left[r^{2} \mathrm{e}^{2 y}+p^{*} \omega\left(\omega+\frac{2}{3}\right)\right]\right\}^{-1},
$$

where $T_{\mathrm{m}}$ denotes the melting temperature.

\section{The Curie temperature calculations}

The Curie temperature is usually assumed as proportional to the exchange integral and the qualitative $T_{\mathrm{C}}(p)$ dependence is nearly independent of the theoretical approach [5]. Taking this fact into consideration we can assume that

$$
\frac{T_{\mathrm{C}}(p)}{T_{\mathrm{C}}\left(p_{0}\right)}=\frac{J\left(R(p), T_{\mathrm{C}}(p)\right)}{J\left(R\left(p_{0}\right), T_{\mathrm{C}}\left(p_{0}\right)\right)},
$$

where $J\left(R(p), T_{\mathrm{C}}(p)\right)$ denotes the exchange integral dependent on the pressure due to its dependence on the distance $R\left(p, T_{\mathrm{C}}(p)\right)$ between two interacting spins. 
The exchange integral dependence on $R$ is discussed in connection with the structure of interactions in amorphous materials [2]. The excellent fitting procedure for the function $J(R)$ is considered by Radomska and Balcerzak [6] for EuTe structure. Taking into account the analogy and profiting the experience of previous calculations $[2,6]$ we can see that the description of the exchange integral in terms of the correlation functions seems to be very convenient, particularly, in the Curie temperature region where we can write

$$
J(R(p))=J_{0} \mathrm{e}^{-\frac{R(p)-R_{0}}{\xi}}
$$

with the correlation range $\xi\left(p, T_{\mathrm{C}}(p)\right)$.

The quantitative agreement of our calculations with experimental data can be obtained by means of an appropriate choice of the parameter $\xi=n R_{0}$ for $n \neq 1$. It is describing the exchange integral dependence on the distance $R\left(p, T_{\mathrm{C}}(p)\right)$ between two interacting spins in terms of the mean square displacements of atoms from their positions of equilibrium calculated by means of the pseudoharmonic approach. For this reason we fit the results presented by $(a)$ in Fig. 1A to the experimental points (b) described by the curve $(c)$ in Fig. 1A. The sufficient agreement of the results is achieved by the parameter $n$ whose shape is shown in Fig. 1B.

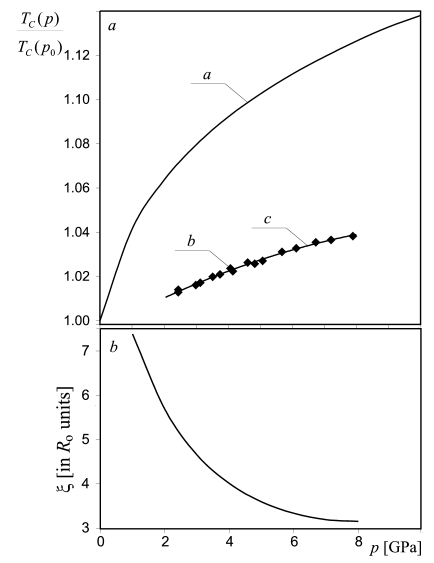

Fig. 1. (A) The pressure dependence of the Curie temperature $T_{\mathrm{C}}(p) / T_{\mathrm{C}}\left(p_{0}\right)$ for $\mathrm{Ni},(a)$ calculated for $\xi=1$, (b) the experimental points [4], (c) fitted to the experimental points by $\xi=\xi(p) . \quad\left(T_{\mathrm{m}}=1728 \mathrm{~K}, T_{\mathrm{C}}\left(p_{0}\right)=\right.$ $627 \mathrm{~K}, R_{0}=3.52 \AA$.) (B) The pressure dependence of the correlation range $\xi$ for the spatial distribution of the exchange integral calculated by the best fit to experimental data.

Substituting (5) to (4) we obtain the self-consistent equation with respect to the variable $T_{\mathrm{C}}(p) / T_{\mathrm{C}}\left(p_{0}\right)$ in the form

$$
\frac{T_{\mathrm{C}}(p)}{T_{\mathrm{C}}\left(P_{0}\right)}=\left[\exp \left(-\left(\frac{R\left(p, T_{\mathrm{C}}(p)\right)}{R\left(p_{0}, T_{\mathrm{C}}\left(p_{0}\right)\right)}-1\right)\right)\right]^{\frac{1}{n}},
$$

whose numerical solution obtained with respect to fitting parameter $n$ shown in Fig. $1 \mathrm{~B}$ allows us to describe the pressure dependence of the Curie temperature.
It is worthwhile to remark that the considerations leading to the equation of state are valid for nonmagnetic materials. The equation can be used for ferromagnets when we describe it in the paramagnetic phase, i.e. $T \geq T_{\mathrm{C}}$ so that it is sufficient for the purposes of the present paper. Below $T_{\mathrm{C}}(p)$ the model includes the coupling between lattice and magnetic component properties. We need then an additional term in equation of state proportional to $J \frac{\partial J(R)}{\partial R}\left\langle S^{z}\right\rangle^{2}$ and the proper correction of the formulae for $y$ parameter simultaneously where $y$ corresponds to the mean square displacement of atoms under the pressure.

\section{Conclusions}

We treat the relation $T_{\mathrm{C}}=T_{\mathrm{C}}(p)$ shown as $(a)$ in Fig. $1 \mathrm{~A}$ as the reference calculations obtained in terms of the pseudoharmonic approximation.

First of all we test the equation of state given in the paramagnetic phase $\left(T \geq T_{\mathrm{C}}\right)$ by means of its relations to the experimental results.

The pressure influence on the Curie temperature depends on the exchange integral with respect to the distance $R(p)$ between two interacting spins. The method needs to know the relation $J=J(p, R(p))$ which can be evaluated by means of the fitting of the exchange integral with respect to the correlation range $\xi=n R_{0}, n=n(p)$ (Fig. 1B). From the physical point of view the obtained result means that the shape of the exchange integral depends on the pressure by two ways: (1) indirectly, via the equation of state and (2) directly, via the influence of the pressure on the elementary cell lattice constant when the electron density connected with the lattice is taken into account. This behaviour is explained as the result of a competition between the decrease in the local magnetic moment and the increase in the magnetic exchange integral as function of rising pressure [5]. The equivalent description presented in this paper corresponds to the interpretation connected with the correlation length behaviour. The fitting $\xi$ in this case leads to $\xi$ which is independent of $p$, that means, it is equivalent to the condition $\partial T_{\mathrm{C}}(p, R(p)) / \partial p=0$ recently discussed in the literature (see, e.g. [5]).

\section{References}

[1] N.M. Plakida, T. Siklós, Phys. Status Solidi B 33, 103 (1969); ibid., 33, 113 (1969); T. Siklós, Acta Phys. Hung. 30, 181 (1971); ibid., 30, 193 (1971); 30, 301 (1971).

[2] S.I. Sorokov, R.R. Levitskii, T.M. Verkholyak, Phys. Status Solidi B 211, 759 (1999).

[3] L. Wojtczak, J. Rutkowski, Š. Zajac, Acta Phys. Superficierum 10, 63 (2008).

[4] J.M. Legger, C. Loners-Susse, B. Vodar, Phys. Rev. B 6, 4250 (1972).

[5] F. Körmann, A. Dick, T. Hickel, J. Neugebauer, Phys. Rev. B 79, 184406 (2009).

[6] A. Radomska, T. Balcerzak, Phys. Status Solidi B 225, 229 (2001). 